YITP-99-52

\title{
Non-commutative Unification in Brane World
}

\author{
Zurab Kakushadze* \\ C.N. Yang Institute for Theoretical Physics \\ State University of New York, Stony Brook, NY 11794
}

(October 9, 1999)

\begin{abstract}
We point out that in (open) string compactifications with non-zero NS-NS $B$-field we can have large Kaluza-Klein thresholds even in the small volume limit. In this limit the corresponding gauge theory description is in terms of a compactification on a non-commutative space (e.g., a torus or an orbifold thereof). Based on this observation we discuss a brane world scenario of noncommutative unification via Kaluza-Klein thresholds. In this scenario, the unification scale can be lowered down to the TeV-range, yet the corresponding compactification radii are smaller than the string length. We discuss a potential application of this scenario in the context of obtaining mixing between different chiral generations which is not exponentially suppressed - as we point out, such mixing is expected to be exponentially suppressed in certain setups with large volume compactifications. We also point out that T-duality is broken by certain non-perturbative twisted open string sectors which are supposed to give rise to chiral generations, so that in the case of a small volume compactification with a rational $B$-field we cannot T-dualize to a large volume description. In this sense, the corresponding field theoretic picture of unification via Kaluza-Klein thresholds in this setup is best described in the non-commutative language.
\end{abstract}

Typeset using REVTEX

*E-mail: zurab@insti.physics.sunysb.edu 
With the advent of D-branes [1] it has become evident that there might exist a logical possibility where the Standard Model gauge and matter fields reside inside of $p \leq 9$ spatial dimensional $p$-branes (or a set of overlapping branes), while gravity lives in a larger (10 or 11) dimensional bulk of space-time. This brane world picture円 a priori appears to be a viable scenario [11]. One of the implications of the brane world scenario is that the string scale $M_{s}$ can be much lower than the four dimensional Planck scale $M_{P}$ [2 [4]. In fact, in [4] it was proposed that $M_{s}$ as well as the fundamental (10 or 11 dimensional) Planck scale $M_{P f}$ can be in the $\mathrm{TeV}$ rangef. The observed weakness of the four dimensional gravitational coupling (that is, the fact that the four dimensional Planck scale $M_{P} \gg M_{s}, M_{P f}$ ) then requires the presence of at least two large $\left(\gg 1 / M_{s}\right)$ compact directions (which are transverse to the branes on which the Standard Model fields are localized). A general discussion of possible brane world embeddings of such a scenario was given in [7,8,11]. In [10] various non-trivial phenomenological issues were discussed in the context of the TeV-scale brane world scenario, and it was argued that this possibility does not appear to be automatically ruled out.

The gauge coupling unification in such a scenario would have to arise in a way drastically different from the usual MSSM unification. In fact, a higher dimensional mechanism for gauge coupling unification in the $\mathrm{TeV}$-scale brane world context was proposed in [5]. In this mechanism the unification scale is lowered compared with the usual MSSM unification scale if one assumes that the Standard Model fields are localized on $p$-branes with $p>3$ (actually, $p=4$ or 5 [12]), and the sizes of $p-3$ compact directions inside of these $p$-branes are somewhat large compared with $1 / M_{s}$. Then the evolution of the gauge couplings above the corresponding Kaluza-Klein (KK) thresholds is no longer logarithmic but power-like [18]. In [12] a TeV-scale Supersymmetric Standard Model (TSSM) was proposed, where the gauge coupling unification indeed occurs via such a higher dimensional mechanism. Moreover, the unification in the TSSM, which occurs in the TeV-range, is as precise at one loop as in the MSSM, and it would also explain why the unification in the MSSM is not an accident. Furthermore, higher loop effects in the TSSM were shown in [12] to be subleading due to the underlying $\mathcal{N}=2$ supersymmetry at the heavy KK levels.

Concrete realizations of the above higher dimensional mechanism for gauge coupling unification in the $\mathrm{TeV}$-scale brane world are expected to be non-trivial. In particular, it is unclear at present how to explicitly embed models such as the TSSM in the string theory framework. This, in turn, is not so surprising as a completely successful embedding of, say, the MSSM in string theory is still lacking. In part this might be due to the lack of necessary

\footnotetext{
${ }^{1}$ For recent developments, see, e.g., [2 12]. The brane world picture in the effective field theory context was discussed in 13,14. Large radius compactifications were originally considered in 15 in the context of supersymmetry breaking.

${ }^{2}$ By the TeV-range we do not necessarily mean $M_{s} \sim 1 \mathrm{TeV}$. In fact, as was argued in [12], the gauge coupling unification constraints seem to imply that $M_{s}$ cannot really be lower than $10-100 \mathrm{TeV}$.

${ }^{3}$ Recently it was proposed in [16] that gravity could also be "localized" and such compact directions might actually not be required. For subsequent works along these lines, see, e.g., [17].
} 
technology for the corresponding model building as such string vacua are expected to be rather non-trivial. Semi-realistic $\mathcal{N}=1$ supersymmetric examples which possess some (but not all) desirable phenomenological features (such as three chiral generations and Standard Model-like gauge groups) were constructed in [6.9] via Type I/orientifold compactificationst. However, as was pointed out in [12], examples of this type do not quite seem to fit in the context of unification via Kaluza-Klein thresholds. In particular, some matter fields (such as some of the chiral generations) must not propagate in the aforementioned extra $p-3$ dimensions or else the corresponding one-loop $\beta$-function coefficients for the power-like running become too positive, and the gauge couplings blow up before they unify. That is, in order to have a successful unification in the TeV-range, we must assume that some (more precisely, at least one) of the chiral generations is localized at a point in the extra $p-3$ dimensions (see the first reference in [12] for details). It is then not difficult to see that such a model cannot be constructed in the perturbative orientifold framework. Indeed, within this framework the only way to have matter localized in a smaller dimensional subspace is if it comes from open string sectors where one of the two ends of the open string is attached to one set of D-branes, while the other end is attached to a different set of D-branes, with these two sets of D-branes overlapping in the subspace of the desired localization. For instance, consider two sets of D5-branes, call them D5 $1^{-}$and D5 $2_{2}$-branes, in $\mathbf{R}^{3,1} \otimes T^{2} \otimes T^{2} \otimes T^{2}$. Let $\mathrm{D} 5_{1}$-branes wrap the first $T^{2}$ while $\mathrm{D} 5_{2}$-branes wrap the second $T^{2}$. Then the $5_{1} 5_{2}$ matter is localized at a point in the compact space where these two sets of branes intersect. Now, let us assume that the Standard Model gauge group comes from the same set of branes, say, D5 $5_{1}$-branes. Note that this is essentially unavoidable or else we would have no unification prediction to begin with - indeed, if different gauge subgroups of the Standard Model gauge group come from different sets of branes (with generically different corresponding compactification volumes), then the gauge couplings for these subgroups at the string scale (which we would eventually like to identify with the unification scale) are not supposed to be the same. So from now on we will assume that all three subgroups of the Standard Model gauge group $S U(3)_{c} \otimes S U(2)_{w} \otimes U(1)_{Y}$ come from the same set of branes, in particular, D $51_{1}$-branes. If this is the case, then at least some of the $5_{1} 5_{2}$ matter corresponding to the Standard Model matter fields would have to be charged under three different gauge factors - $S U(3)_{c} \otimes S U(2)_{w}$ (here we are being overly generous with $U(1)_{Y}$ whose more careful treatment could only worsen the situation) as well as a gauge subgroup of the D5 $5_{2}$-brane gauge group. In particular, left-handed electroweak doublet quarks would have to be such states. This is, however, impossible in the perturbative orientifold framework as there open strings have only two ends, hence only one type of distinct Chan-Paton factors is allowed for a given set of D-branes.

Thus, we must find other possible mechanisms for matter localization which would take us outside of the perturbative orientifold construction. In [12] it was pointed out that an

\footnotetext{
${ }^{4}$ For other recent developments in four dimensional Type I/orientifold compactifications, see, e.g., [19,20].

${ }^{5}$ Here we choose a simple toroidal compactification only for illustrative purposes. In more realistic cases one could equally successfully consider, say, toroidal orbifolds.
} 
adequate framework for such model building might be that of non-perturbative orientifolds examples of which have been recently constructed in 20]. In such vacua, which (generically) are non-perturbative from both Type I and heterotic viewpoints, there are additional twisted open string sectors (which do not possess perturbative orientifold description) giving rise to states charged under, say, the gauge group coming from some set of D5-branes and, at the same time, localized at orbifold fixed points. This is analogous to what happens in perturbative heterotic superstring where some of the states charged under the appropriate subgroups of $\operatorname{Spin}(32) / \mathbf{Z}_{2}$ or $E_{8} \otimes E_{8}$ come from twisted closed string sectors. In fact, this is not just a mere analogy but has more substance as using Type I-heterotic duality one can in certain cases map twisted open string sectors to twisted closed string sectors which have perturbative heterotic description [20]. In more complicated cases where no perturbative description exists in either pictures one can still understand such states using a "hybrid" approach which involves utilizing some geometric aspects of orbifold compactifications.

As we have already mentioned, the matter localized at orbifold fixed points in nonperturbative orientifold compactifications essentially behaves as twisted closed string states in heterotic string theory. In particular, couplings of such twisted open string matter fields localized at orbifold fixed points obey the point-group and space-group selection rules (see, e.g., [21] for details). In the following we will mostly be interested in the space-group selection rules, so let us briefly review their essence using a simple example.

Thus, consider a $T^{2} / \mathbf{Z}_{3}$ orbifold, where the generator $g$ of the orbifold group acts crystallographically on $T^{2}$ by $2 \pi / 3$ rotations. The metric on $T^{2}$ is given by $g_{a b}=(v / \sqrt{3}) e_{a} \cdot e_{b}$, $a, b=1,2$, where $v$ is the volume of $T^{2}$, and $e_{a}$ are the vectors generating the $S U(3)$ root lattice $\Lambda \equiv\left\{n^{a} e_{a} \mid n^{a} \in \mathbf{Z}\right\}$ (note that $e_{1}^{2}=e_{2}^{2}=2$, and $e_{1} \cdot e_{2}=-1$ ). The $\mathbf{Z}_{3}$ twist acts with three fixed points. One of them is located at the origin, $\xi_{0}=0$, while the other two are located (up to immaterial torus identifications) at $\xi_{ \pm}= \pm \sqrt{v / \sqrt{3}} \widetilde{e}^{2}$, where $\widetilde{e}^{a}, a=1,2$, generate the $S U(3)$ weight lattice $\widetilde{\Lambda} \equiv\left\{m_{a} \widetilde{e}^{a} \mid m_{a} \in \mathbf{Z}\right\}$ (note that $\left(\widetilde{e}^{1}\right)^{2}=\left(\widetilde{e}^{2}\right)^{2}=2 / 3$, and $\left.\widetilde{e}^{1} \cdot \widetilde{e}^{2}=1 / 3\right)$. The space-group selection rules imply that, for instance, three-point couplings of twisted fields $T_{A}, T_{B}$ and $T_{C}$, where $A, B, C=0, \pm$ label the corresponding fixed points, are non-vanishing if and only if $A=B=C$ or $A \neq B \neq C \neq A$ [21]. The couplings of twisted fields coming from the same fixed point are unsuppressed. However, the couplings of twisted fields coming from different fixed points are exponentially suppressed. In particular, they are suppressed by an exponential factor $\exp \left(-c v M_{s}^{2}\right)$ [21], where $c$ is a numerical coefficient of order 1 . Thus, in the large volume limit, that is, when $v \gg M_{s}^{-2}$, the "off-diagonal" twisted sector couplings are exponentially small. In the closed string theory language this can be understood from the fact that such couplings come from world-sheet instantons arising from strings wrapping the corresponding 2-cycles in the orbifold. The action of such world-sheet instantons is proportional to the volume $v$ of these two-cycles, hence the above exponential factor. However, we can also understand this from the field theory viewpoint - the overlap of wave-functions for the states localized at different fixed points is exponentially (more precisely, Gaussian-like) suppressed with the distance between the fixed points (which is proportional to $\sqrt{v}$ ). In fact, this way of thinking about this point makes it clear why in non-perturbative orientifolds some of the aspects of twisted open strings such as twisted sector couplings should mimic the corresponding statements in conformal field theory of twisted closed strings.

The above discussion might have interesting phenomenological implications for the uni- 
fication via Kaluza-Klein thresholds. Thus, as was pointed out in [12, to have a meaningful unification prediction we must ensure that higher loops are subleading compared with the leading one-loop threshold corrections to the gauge couplings. This is by no means automatic in these scenarios. Thus, the number of heavy KK modes propagating in loops $N \sim v M_{s}^{2} \gg 1$. The actual loop expansion parameter then is not given by the low energy gauge coupling $\alpha / 4 \pi$. Instead, the loop expansion parameter is $\sim N \alpha / 4 \pi \sim \lambda_{s}$, where $\lambda_{s}$ is the string expansion parameter (which in the conventions of [22] is related to the string coupling $g_{s}$ via $\lambda_{s}=g_{s} / 4 \pi$ ). In fact, even though the low energy gauge coupling $\alpha$ is relatively small, the loop expansion parameter $\lambda_{s} \sim 1$. Then without any additional cancellations higher loop corrections to the gauge couplings would be as large as the one-loop threshold contribution, hence lack of unification prediction.

There is, however, a way to make unification via KK thresholds predictive. Thus, as was pointed out in [12, if we consider $\mathcal{N}=1$ supersymmetric theories, the heavy KK modes have $\mathcal{N}=2$ supersymmetry in the sense that their spectrum as well as their couplings to each other are $\mathcal{N}=2$ supersymmetric. Then non-renormalization properties of $\mathcal{N}=2$ gauge theories beyond one loop result in additional suppression of higher loop corrections to the gauge couplings due to (partial) $\mathcal{N}=2$ cancellations so that higher loop effects are subleading compared with the leading one-loop threshold correction. This renders unification via KK thresholds predictive.

A way to obtain $\mathcal{N}=1$ supersymmetric gauge theories with $\mathcal{N}=2$ supersymmetry at heavy KK levels is via certain orbifold compactifications. In particular, one can consider generalized Voisin-Borcea orbifolds defined as

$$
\mathcal{M}=\left(T^{2} \otimes \mathrm{K} 3\right) / \mathbf{Z}_{M}
$$

where the generator $g$ of $\mathbf{Z}_{M}$ acts crystallographically on $T^{2}$ by $2 \pi / M$ rotation $g z_{1}=\omega z_{1}$, and it is a symmetry of K3 acting on the holomorphic 2 -form $\Omega_{2}$ on K3 via $g \Omega_{2}=\omega^{-1} \Omega_{2}$. Here $z_{1}$ is the complex coordinate parametrizing $T^{2}$, and $\omega \equiv \exp (2 \pi i / M)$. Note that $M$ can only take values $M=2,3,4,6$ (or else the action of $g$ on $T^{2}$ would not be crystallographic). The Calabi-Yau three-fold $\mathcal{M}$, which is an elliptic fibration of $T^{2}$ over the base $\mathcal{B} \equiv \mathrm{K} 3 / \mathbf{Z}_{M}$, has $S U(3)$ holonomy, so, say, Type I compactified on $\mathcal{M}$ has $\mathcal{N}=1$ supersymmetry in four dimensions (provided that the corresponding tadpoles can be canceled). Now consider D5-branes wrapping the fibre $T^{2}$. The four dimensional massless modes of the gauge theory in the world-volume of the D5-branes have $\mathcal{N}=1$ supersymmetry. However, the heavy KK modes on $T^{2}$ actually come in $\mathcal{N}=2$ supersymmetric multiplets, and, as was shown in [12], interactions involving only heavy $\mathrm{KK}$ modes are also $\mathcal{N}=2$ supersymmetric. This is, therefore, a setup where we could consider unification via KK thresholds.

As we have already mentioned, at least one of the chiral generations must come from the twisted open string sectors $\left[\right.$ localized at fixed points of $T^{2} / \mathbf{Z}_{M}$. Now, let us assume for

\footnotetext{
${ }^{6}$ Note that in scenarios with unification in the TeV-range the volume of $\mathrm{K} 3$ must be large in the string units in order for the observed four dimensional Planck scale to be much larger than the string scale, the latter being identified with the TeV-range unification scale.

${ }^{7}$ As was pointed out in [20], non-perturbative twisted open string sectors arise in $\mathbf{Z}_{M}$ orbifolds
} 
a moment that all chiral generations arise from such twisted open string sectors. Then, as we pointed out above, couplings between different generations would be exponentially suppressed in the limit of large volume $T^{2}$. That is, mixing between different generations would be unacceptably small contradicting the observed CKM matrix. Thus, at least naively, having large threshold corrections seems incompatible with the desired mixing between different chiral generations if all of them arise in $\mathbf{Z}_{M}$ twisted open string sectors.

A priori we could assume that two of the chiral generations arise in untwisted (with respect to the action of the $g$ twist ${ }^{8}$ ) open string sectors while one generation comes from a twisted open string sector to possibly avoid the above difficulty with small mixing. However, in certain cases it might be desirable to have all three generations coming from twisted open string sectors. For instance, in the TSSM to ensure proton stability in [12] a generationblind discrete $\mathbf{Z}_{3} \otimes \mathbf{Z}_{3}$ gauge symmetry was proposed. This discrete symmetry can be identified with the corresponding orbifold discrete symmetry, which would imply that all three generations would have to arise in $\mathbf{Z}_{M}$ twisted open string sectors $(M=3$ in this case - see the second reference in [12] for details). The question we would like to address next is whether we can have large threshold corrections with all three generations coming from fixed points in the fibre $T^{2}$.

Here we would like to point out that there indeed seems to exist such a setup. In particular, in the above discussion we have made an implicit assumption that the untwisted NS-NS $B$-field in the directions of $T^{2}$ is trivial. Let us, however, consider the case where we have a non-trivial $B$-flux on the fibre $T^{2}$. In this case the Kaluza-Klein spectrum is modified, and the behavior of the corresponding threshold corrections with the volume of $T^{2}$ can be quite different from the case without the $B$-field. In particular, as we will see in a moment, if there is a non-trivial $B$-flux, we can have large threshold corrections even if the volume of $T^{2}$ is small compared with $\alpha^{\prime}=M_{s}^{-2}$.

For the reasons which will become clear in the following, we will mostly be interested in the $\mathbf{Z}_{3}$ and $\mathbf{Z}_{6}$ cases, where the fibre $T^{2}$ must have the appropriate $\mathbf{Z}_{3}$ symmetry. However, for illustrative purposes we will discuss the case of a square torus $T^{2}=S^{1} \otimes S^{1}$ (with identical radii of the two circles) with a non-zero $B$-field as this simple example captures all the key points relevant for the subsequent discussions. Thus, the metric on $T^{2}$ and the $B$-field are given by

$$
G_{a b}=\left(\begin{array}{cc}
v & 0 \\
0 & v
\end{array}\right), \quad B_{a b}=\left(\begin{array}{cc}
0 & b \\
-b & 0
\end{array}\right)
$$

where $v$ is the volume of $T^{2}$, and the $B$-field is defined up to unit shifts $b \rightarrow b+1$. Without the $B$-field the metric felt by the open string sector is simply $G_{a b}$. In particular, the KK modes coming from D5-branes wrapping $T^{2}$ have mass squared $M_{\mathbf{m}}^{2} \sim G^{a b} m_{a} m_{b}$ (here

with $M=3,4,6$, but not with $M=2$.

\footnotetext{
${ }^{8}$ Note that in the case of orbifold K3 these generations could still come from open string sectors twisted with respect to another twist $\theta$ which is the generator of the K3 orbifold group $\mathbf{Z}_{M^{\prime}}$ (here $\left.\mathrm{K} 3=T^{4} / \mathbf{Z}_{M^{\prime}}\right)$.
} 
$\left.\mathbf{m} \equiv\left(m_{1}, m_{2}\right)\right)$, where $G^{a b}$ is the inverse of $G_{a b}$. However, if the $B$-field is non-trivial, the metric felt by open strings is given by (we are working in the units $2 \pi \alpha^{\prime}=1$ ) f

$$
\mathcal{G}_{a b}=G_{a b}-B_{a c} G^{c d} B_{d b}=\left(\begin{array}{cc}
v+b^{2} / v & 0 \\
0 & v+b^{2} / v
\end{array}\right)
$$

and, in particular, the KK modes have mass squared $M_{\mathbf{m}}^{2} \sim \mathcal{G}^{a b} m_{a} m_{b}$, where $\mathcal{G}^{a b}=\left(v /\left(v^{2}+\right.\right.$ $\left.\left.b^{2}\right)\right) \delta_{a b}$ is the inverse of $\mathcal{G}_{a b}$. Note that if the volume of $T^{2}$ is small (that is, $v \ll \alpha^{\prime}$ ), in the case with the $B$-field (such that $b \sim 1$ ) we have light KK modes with masses of order $\sqrt{v} / b \ll M_{s}$. This is to be contrasted with the case without the $B$-field where the lightest massive KK mode has a mass of order $1 / \sqrt{v} \gg M_{s}$. Thus, in the case with the $B$-field we have a large number of massive KK modes below the cut-off (that is, string) scale $M_{s}$ which contribute into the threshold corrections to the gauge couplings. In fact, the leading one-loop threshold contribution is of order $N^{\prime} \sim b^{2} \alpha^{\prime} / v \gg 1$, the number of massive $\mathrm{KK}$ modes below the cut-off $M_{s}$.

The above discussion can be straightforwardly generalized to the case of the most general $T^{2}$. In fact, we are going to give the general expressions for the one-loop threshold corrections to the gauge couplings in the small volume limit in the presence of a non-trivial $B$-flux. First let us consider D5-branes wrapping $T^{2}$ with the space transverse to the branes being K3. The corresponding gauge theory has $\mathcal{N}=2$ supersymmetry at both massless and massive KK levels. Let the gauge group be $\bigotimes_{i} G_{i}$ with the low energy gauge couplings $\alpha_{i}(\mu)$ (here $\mu$ is the energy scale at which the gauge couplings are measured). The gauge couplings in this case are renormalized only at one loop, and are given by:

$$
\alpha_{i}^{-1}(\mu)=\alpha^{-1}+\frac{\widetilde{b}_{i}}{2 \pi} \ln \left(\frac{M_{s}}{\mu}\right)+\widetilde{\Delta}_{i},
$$

where $\alpha \equiv g_{s} / 2 \nu$ (we will define $\nu$ in a moment) is the unified gauge coupling at the string scale $M_{s}, \widetilde{b}_{i}$ are the corresponding $\mathcal{N}=2$ one-loop $\beta$-function coefficients, and the threshold corrections corresponding to the massive KK modes are given by (here we assume that $\nu \gg 1)$

$$
\widetilde{\Delta}_{i}=\frac{\widetilde{b}_{i}}{4 \pi} \xi^{2} \nu-\frac{\widetilde{b}_{i}}{4 \pi} \ln (\nu)+\mathcal{O}(1) .
$$

Here $\xi$ parametrizes the subtraction scheme dependence. In particular, the infra-red (IR) cut-off in the loop integrals is taken as $\xi \mu$, while the ultra-violet (UV) cut-off is taken as $\xi M_{s}$.

In the above expressions the quantity $\nu$ is defined as

$$
\nu \equiv\left(M_{s} / 2 \pi\right)^{2} \sqrt{\operatorname{det}\left(\mathcal{G}_{a b}\right)}=\left(M_{s} / 2 \pi\right)^{2} \operatorname{det}\left(G_{a b}+\left(2 \pi \alpha^{\prime}\right) B_{a b}\right) / \sqrt{\operatorname{det}\left(G_{a b}\right)} .
$$

\footnotetext{
${ }^{9}$ For a recent discussion of D-branes wrapped on tori with non-zero $B$-flux using the boundary state formalism, see 23.
} 
Note that in the small volume limit $v \ll \alpha^{\prime}$ we have $\nu \approx b^{2}\left(\alpha^{\prime} / v\right)$. In the large volume limit the $B$-field does not play any important role, and we recover the usual result $\nu \approx\left(M_{s} / 2 \pi\right)^{2} v$ [12.

Next, let us consider the case where D5-branes are wrapping the fibre $T^{2}$ in the compactification on a generalized Voisin-Borcea orbifold (11). In this case we have $\mathcal{N}=1$ supersymmetry at the massless level, while the heavy KK modes are still $\mathcal{N}=2$ supersymmetric. The gauge couplings are now given by

$$
\alpha_{i}^{-1}(\mu)=\alpha^{-1}+\frac{b_{i}}{2 \pi} \ln \left(\frac{M_{s}}{\mu}\right)+\Delta_{i},
$$

where $b_{i}$ are the $\mathcal{N}=1$ one-loop $\beta$-function coefficients for the massless modes. The one-loop threshold corrections in this case are given by

$$
\Delta_{i}=\widetilde{\Delta}_{i} / M
$$

where $M$ is the order of the generalized Voisin-Borcea orbifold.

Thus, in the presence of the $B$-field we can have large threshold corrections to the gauge couplings, so that unification via KK thresholds can occur at low scales as in [5, 12]. The key point here, however, is that this is also the case for compactifications on small (rather than only large) tori (or, more precisely, orbifolds thereof). That is, the mixing between the chiral generations coming from (non-perturbative) twisted open string sectors localized at the corresponding orbifold fixed points need no longer be exponentially suppressed.

The above discussion of D5-branes wrapping $T^{2}$ with non-zero $B$-flux has one loose end which we would like to tie up next. In particular, so far we have been assuming that the the $B$-field is taking generic values. However, at special values of the $B$-field, namely, at certain rational values of $b$, we can perform a T-duality transformation which maps a small volume $T^{2}$ to a large volume "dual" torus - for a recent comprehensive discussion and references, see [24]. The corresponding KK threshold corrections in the case of a large volume $T^{2}$ are still expected to be large, and, as we will see in a moment, they are identical in this Tdual description to those in the language of the original small volume torus. However, at least naively, there might seem to be a puzzle as for a large volume $T^{2}$ we expect twisted open string sector states to have exponentially small off-diagonal couplings, which is not the same as what we have concluded for a small $T^{2}$ compactification. As we will explain in a moment, there is no puzzle here as T-duality is a good symmetry in the perturbative (from the orientifold viewpoint) open string sectors but is broken in the non-perturbative twisted open string sectors whenever we have the corresponding off-diagonal couplings.

The reason why the above point is especially important is that if we consider Type I/orientifold compactifications, the $B$-field cannot take arbitrary values but must be quantized. Indeed, let $\Omega$ be the orientifold action interchanging the left- and right-movers on the closed string world-sheet. The NS-NS $B$-field is antisymmetric under the action of $\Omega$.

\footnotetext{
${ }^{10}$ Here we ignore heavy string oscillator thresholds which are $\mathcal{O}(1)$ in $\mathcal{N}=1$ theories but still non-vanishing. Note that in $\mathcal{N}=2$ theories such thresholds are absent - see [12 for details and references.
} 
Taking into account that it is defined only up to unit shifts, it is then clear that $b$ can only take two values consistent with the orientifold action: $b=0$ and $b=1 / 2$. In fact, the massless closed string states corresponding to the $B$-field moduli are projected out by the orientifold action which implies that $b$ cannot take continuous values. However, it can take the above quantized values, so from now on we will focus on backgrounds with $b=1 / 2$. (For more details on orientifolds with non-zero B-field, see, e.g., 25 $\square$.)

For the sake of simplicity we will consider the case of a square torus with the metric and the $B$-field given by (2). We will assume that $b=1 / k$, where $k \in \mathbf{N}-\{1\}$. In this case, if we start from a torus with small volume, via a T-duality transformation we can map it to a torus with large volume. To describe this T-duality transformation, let us introduce the following matrix (here we are working in the units $2 \pi \alpha^{\prime}=1$, and we are closely following the discussion in [28,24):

$$
E_{a b} \equiv G_{a b}+B_{a b}
$$

The T-duality group in the case of $T^{2}$ is $S O(2,2, \mathbf{Z})$ whose elements can be described in terms of $4 \times 4$ matrices

$$
\left(\begin{array}{ll}
\alpha & \beta \\
\gamma & \delta
\end{array}\right)
$$

\footnotetext{
${ }^{11}$ In [25] it was assumed that the closed strings that couple to D-branes wrapping, say, a 2-torus with $b=1 / 2$ satisfy the "no momentum flow" condition in the directions of $T^{2}$, that is, these states have left- and right-moving momenta satisfying $P_{L a}=-P_{R a}$. This is only one of the two a priori available choices. In particular, the second choice corresponds to imposing the condition $P_{L a}=-\mathcal{R}_{a}{ }^{b} P_{R b}$, where the matrix $\mathcal{R}$ is given by $\mathcal{R} \equiv E^{T} E^{-1}$ (see the next paragraph in the main text for notations). In the former case the closed string states that couple to D-branes satisfy $m_{a}-B_{a b} n^{b}=0$, which, in particular, implies that the winding numbers $n^{a}$ must be even. This, as explained in [25], leads to the rank reduction for the Chan-Paton gauge group. On the other hand, if we impose the condition $P_{L a}=-\mathcal{R}_{a}{ }^{b} P_{R b}$, then the corresponding closed strings satisfy $m_{a}=0$ with arbitrary winding numbers $n^{a}$, and the metric felt by open strings is $\mathcal{G}_{a b}$. In this case the rank of the Chan-Paton gauge group would not be reduced. In the context of perturbative orientifolds, however, there is an additional consistency condition one needs to impose, namely, that the branes and orientifold planes couple to each other in a consistent fashion. This would require that the orientifold plane couples to the closed string states whose mass squared $M_{\mathbf{n}}^{2} \sim \mathcal{G}_{a b} n^{a} n^{b}$. On the other hand, in the loop channel for the Klein bottle the orientifold action implies that the corresponding momentum states have mass squared $M_{\mathbf{m}}^{2} \sim G^{a b} m_{a} m_{b}$. Note that since the loop channel is related to the tree channel via the $t \rightarrow 1 / t$ modular transformation, the consistency requires that the metric $G^{a b}$ be the inverse of $\mathcal{G}_{a b}$, which is only the case for the zero $B$-field. Thus, the aforementioned second choice does not seem to be consistent in the context of perturbative orientifolds with non-zero $B$-field. However, in the framework of non-perturbative orientifolds, which we are interested in here, this need not be the case. In particular, we will assume that the $B$-field is quantized, and the metric felt by open strings is $\mathcal{G}_{a b}$. Such a setup is conceivable in F-theory where we do not have orientifold planes but the B-field must be appropriately quantized [26,27.
} 
where $\alpha, \beta, \gamma, \delta$ are $2 \times 2$ matrices with integer entries, and satisfy the following constraints:

$$
\begin{aligned}
& \gamma^{T} \alpha+\alpha^{T} \gamma=0 \\
& \delta^{T} \beta+\beta^{T} \delta=0 \\
& \gamma^{T} \beta+\alpha^{T} \delta=I .
\end{aligned}
$$

Here the superscript $T$ stands for transposition, and $I$ denotes the $2 \times 2$ identity matrix. The above T-duality element acts on $E_{a b}$ as follows:

$$
E \rightarrow E^{\prime}=(\alpha E+\beta)(\gamma E+\delta)^{-1}
$$

Note that we can write $E=v I+b \Sigma$, where $\Sigma$ is the $2 \times 2$ antisymmetric matrix with $\Sigma_{12}=1$. Next, consider the T-duality transformation with (recall that $k=1 / b$ )

$$
\alpha=I, \quad \beta=0, \quad \gamma=k \Sigma, \quad \delta=I .
$$

We will denote this T-duality transformation by $P$. The corresponding matrix $E^{\prime}$ is given by:

$$
E^{\prime}=\frac{b^{2}}{v} I-b \Sigma
$$

Note that the T-duality transformation $P$ amounts to $v \rightarrow b^{2} / v, b \rightarrow-b$. In particular, a small volume torus with the $B$-field is mapped to a large volume torus with the opposite $B$-field.

So far we have not said anything about what happens to D5-branes under the T-duality transformation $P$. As we will see in a moment, D5-branes wrapping the original $T^{2}$ transform into D5-branes wrapping the dual torus under the transformation $P$. This is consistent with the discussion after (6), in particular, the threshold corrections computed on the original small volume torus are identical to those on the dual large volume torus, which can be seen by recalling that the T-duality transformation $P$ acts as $v \rightarrow\left(2 \pi \alpha^{\prime} b\right)^{2} / v$ (here we have restored the appropriate factors of $\left.2 \pi \alpha^{\prime}\right)$.

To explicitly see that the transformation $P$ maps D5-branes to D5-branes (and not, say, D3-branes), let us analyze the structure of $P$ in terms of the familiar T-duality transformations $S$ and $T$. The $S$-transformation corresponds to taking $\alpha=0, \beta=I, \gamma=I$ and $\delta=0$, and amounts to mapping a 2-torus with metric $G$ and zero $B$-field to another 2-torus (with zero $B$-field) whose metric is given by the inverse of $G$. This is just the usual " $R \rightarrow 1 / R$ " type of T-duality transformation. On the other hand, the $T$-transformation corresponds to taking $\alpha=I, \beta=\Sigma, \gamma=0$ and $\delta=I$, and amounts to unit shifts of the $B$-field (but does not affect the metric on $T^{2}$ ). It is not difficult to see that the transformation $P$ can be written as

$$
P=S T^{k} S
$$

Thus, under the first $S$-transformation D5-branes are mapped to D3-branes, the subsequent $T$-transformations do not affect the dimensionality of branes, and the last $S$-transformations maps D3-branes back to D5-branes. 
As we see, in the T-dual picture we have D5-branes wrapping a large volume torus. We must then explain how come the twisted open string sector states localized at the fixed points of the corresponding $\mathbf{Z}_{M}$ orbifold of $T^{2}$ have unsuppressed off-diagonal couplings in the original picture while in the dual picture we expect them to be exponentially small. To understand this point better, let us review some facts about twisted open string states in non-perturbative orientifolds discussed in detail in [20]. First, non-perturbative twisted open string states do not arise in the $\mathbf{Z}_{2}$ twisted sectors. There are twisted open string states arising in the $\mathbf{Z}_{4}$ twisted sectors, that is, $\Omega g$ and $\Omega g^{-1}$ twisted open string sectors, where $g$ is the generator of $\mathbf{Z}_{4}$. However, only one of the two fixed points of $g$ acting on $T^{2}$ gives rise to such states, namely, that at the origin. As pointed out in [20], the other fixed point does not give rise to non-perturbative twisted open string states as there is a twisted half-integer $B$-flux stuck inside of the corresponding $\mathbf{P}^{1}$ along the lines of [29]. In fact, this is consistent with the fact that in the $\mathbf{Z}_{4}$ case $\mathrm{T}$-duality on the fibre $T^{2}$ is a good symmetry of the corresponding background, which is unbroken by the non-perturbative twisted open string states [20]. Thus, in the $\mathbf{Z}_{4}$ case we have no aforementioned puzzle with the offdiagonal couplings of twisted open string states as there are no such couplings to begin with - all twisted open string states come from the fixed point at the origin of $T^{2}$. Thus, using T-duality in this case we can map the original compactification with a small volume $T^{2}$ to the dual compactification with a large volume 2-torus.

However, the situation is quite different in the $\mathbf{Z}_{3}$ and $\mathbf{Z}_{6}$ cases. We will focus on the $\mathbf{Z}_{3}$ case as the $\mathbf{Z}_{6}$ case is similar. Thus, in the $\mathbf{Z}_{3}$ case we have twisted open string states arising at all three fixed points of $T^{2} / \mathbf{Z}_{3}$, and they do possess off-diagonal couplings. In the original small volume torus compactification these are unsuppressed, whereas in the dual large volume torus compactification they would be exponentially suppressed, which would lead to a puzzletz. The resolution of this point is that, as was originally pointed out in [20], T-duality is not a good symmetry of this background. In particular, it is broken by non-perturbative twisted open string sectors (albeit the perturbative (from the orientifold viewpoint) open string sectors do possess the corresponding T-duality symmetry). This can be seen from, say, the corresponding explicit $\mathbf{Z}_{6}$ examples constructed in [20]. Thus, for instance, if we consider the non-perturbative orientifold corresponding to Type I on $\mathrm{K} 3=T^{4} / \mathbf{Z}_{6}$, it contains both D9- and D5-branes (the former wrap K3, while the latter are transverse to K3 and are located at the same orientifold point at the origin of K3). The perturbative (from the orientifold viewpoint) 99 and 55 open sting sectors have identical spectra as they should - T-duality is a good symmetry in these sectors. However, the twisted 99 and 55 open string spectra are different. In fact, the correctness of these spectra follows from Type I-heterotic duality plus anomaly cancellation requirements. In particular, had the twisted 99 and 55 open string spectra been the same, the six dimensional gravitational anomalies would not have canceled. Thus, T-duality is indeed broken by twisted open string

\footnotetext{
${ }^{12}$ Note that there is no such puzzle in the closed string sector as the closed string states (both twisted and untwisted) do not possess Yukawa or quartic scalar couplings - such couplings are absent in the parent oriented closed string compactification with $\mathcal{N}=2$ supersymmetry. However, in the twisted open string sectors we do have the corresponding couplings so that one does need to address this issue.
} 
sectors. The physical reason for this can be intuitively understood by noticing that the aforementioned T-duality transformation $S$ in the orientifold language maps $\Omega$ to $\Omega R(-1)^{F_{L}}$, where $R$ is the reflection on the fibre $T^{2}\left(R z_{1}=-z_{1}\right)$, and $F_{L}$ is the left-handed space-time fermion number operator. The two of the three $T^{2} / \mathbf{Z}_{3}$ fixed points $\xi_{ \pm}$(that is, those not located at the origin) are not invariant under the action of the reflection $R$, hence the lack of T-duality symmetry in such backgrounds ${ }^{\mathrm{T}}$. The upshot of this discussion is that we cannot use T-duality transformation $P$ to map a small volume $T^{2}$ compactification to a large volume dual 2-torus compactification, and this avoids the puzzle with the off-diagonal couplings between the twisted open string states in these backgrounds. Practically, this means that if we start from a compactification with a small volume fibre $T^{2}$, we are stuck with this description where the $B$-field plays a crucial role for unification via Kaluza-Klein thresholds.

Before we finish our discussion, we would like to make the following remark. First, we can ask if we could understand the KK thresholds without appealing to string theory at all - after all, computation of KK thresholds without the $B$-field can be carried out without any reference to string theory except when discussing the UV cut-off, which is chosen to be $\sim M_{s}$. In the case with non-zero $B$-field we can also give a purely field theoretic description. In particular, in the regime of a small volume $T^{2}$ with the $B$-field we have a description in terms of the corresponding gauge theory on a non-commutative torus (see [24] and

${ }^{13} \mathrm{~A}$ breakdown of T-duality due to non-perturbative effects was also argued in a different context in [30]. It would be interesting to understand if there is any relation between the results of [30] and the earlier observations in 20].

${ }^{14}$ As we have argued above, in the $\mathbf{Z}_{3}$ and $\mathbf{Z}_{6}$ cases we would have no choice but to stick to the non-commutative description if we chose to work within the field theory language. In the $\mathbf{Z}_{4}$ case, however, T-duality is a good symmetry of the corresponding background, and we could rewrite the theory in the commutative language. More precisely, the corresponding gauge theory is still described in terms of the compactification on a non-commutative torus (since the $B$-field is still non-zero in the T-dual picture), but it is well approximated by the commutative description as the volume of the torus is large (this is, in a sense, equivalent to the zero string slope limit $\alpha^{\prime} \rightarrow 0$ ). At any rate, the $\mathbf{Z}_{3}$ and $\mathbf{Z}_{6}$ cases might be appealing for solving the proton stability as well as neutrino mass problems in the TSSM (where we have three chiral generations), see the second reference in [12] for details. On the other hand, these discrete symmetries might be relevant (see the second reference in [31]) for gauge coupling unification in the recently proposed TSSM4 model [31], where we have four chiral generations, and the electroweak Higgs is identified with a fourth generation slepton. Another potential use for such discrete symmetries might be within the context of (discrete) flavor gauge symmetries proposed in [32] as a solution to the problem of flavor changing neutral currents (for other related works, see, e.g., 33]). In fact, it is likely that such (discrete) flavor symmetries would require that the corresponding chiral generations come from non-perturbative twisted open string sectors as it is difficult to imagine how they would arise in the perturbative orientifold framework. 
references therein)巴 with the UV cut-off at $\sim M_{s}$. This is the motivation for the title of this paper.

I would like to thank Martin Roček and Tom Taylor for discussions. This work was supported in part by the National Science Foundation. I would like to thank the string theory group at Harvard University for their kind hospitality while this work was completed. I would also like to thank Albert and Ribena Yu for financial support.

\footnotetext{
${ }^{15}$ Some aspects of non-commutative extra large dimensions (that is, those transverse to the branes on which the Standard Model fields are localized) have been recently discussed in [34.
} 


\section{REFERENCES}

[1] J. Polchinski, Phys. Rev. Lett. 75 (1995) 4724.

[2] E. Witten, Nucl. Phys. B471 (1996) 135.

[3] J. Lykken, Phys. Rev. D54 (1996) 3693.

[4] N. Arkani-Hamed, S. Dimopoulos and G. Dvali, Phys. Lett. B429 (1998) 263; Phys. Rev. D59 (1999) 086004.

[5] K.R. Dienes, E. Dudas and T. Gherghetta, Phys. Lett. B436 (1998) 55; Nucl. Phys. B537 (1999) 47; hep-ph/9807522.

[6] Z. Kakushadze, Phys. Lett. B434 (1998) 269; Nucl. Phys. B535 (1998) 311; Phys. Rev. D58 (1998) 101901.

[7] I. Antoniadis, N. Arkani-Hamed, S. Dimopoulos and G. Dvali, Phys. Lett. B436 (1998) 257.

[8] G. Shiu and S.-H.H. Tye, Phys. Rev. D58 (1998) 106007.

[9] Z. Kakushadze and S.-H.H. Tye, Phys. Rev. D58 (1998) 126001.

[10] N. Arkani-Hamed, S. Dimopoulos and G. Dvali, Phys. Rev. D59 (1999) 086004.

[11] Z. Kakushadze and S.-H.H. Tye, Nucl. Phys. B548 (1999) 180.

[12] Z. Kakushadze, Nucl. Phys. B548 (1999) 205; Nucl. Phys. B552 (1999) 3;

Z. Kakushadze and T.R. Taylor, hep-th/9905137.

[13] V. Rubakov and M. Shaposhnikov, Phys. Lett. B125 (1983) 136;

A. Barnaveli and O. Kancheli, Sov. J. Nucl. Phys. 52 (1990) 576.

[14] G. Dvali and M. Shifman, Nucl. Phys. B504 (1997) 127; Phys. Lett. B396 (1997) 64.

[15] I. Antoniadis, Phys. Lett. B246 (1990) 377.

[16] M. Gogberashvili, hep-ph/9812296; hep-ph/9812365.

[17] L. Randall and R. Sundrum, hep-ph/9905221; hep-th/9906064;

H. Verlinde, hep-th/9906182;

N. Arkani-Hamed, S. Dimopoulos, G. Dvali and N. Kaloper, hep-th/9907209.

[18] T.R. Taylor and G. Veneziano, Phys. Lett. B212 (1988) 147.

[19] M. Berkooz and R.G. Leigh, Nucl. Phys. B483 (1997) 187;

C. Angelantonj, M. Bianchi, G. Pradisi, A. Sagnotti and Ya.S. Stanev, Phys. Lett. B385 (1996) 96;

Z. Kakushadze, Nucl. Phys. B512 (1998) 221; Nucl. Phys. B529 (1998) 157;

Z. Kakushadze and G. Shiu, Phys. Rev. D56 (1997) 3686; Nucl. Phys. B520 (1998) 75;

G. Zwart, Nucl. Phys. B526 (1998) 378;

G. Aldazabal, A. Font, L.E. Ibáñez and G. Violero, Nucl. Phys. B536 (1998) 29;

Z. Kakushadze, G. Shiu and S.-H.H. Tye, Nucl. Phys. B533 (1998) 25;

J. Lykken, E. Poppitz and S.P. Trivedi, Nucl. Phys. B543 (1999) 105;

R. Blumenhagen and A. Wisskirchen, Phys. Lett. B438 (1998) 52;

L.E. Ibáñez, R. Rabadan and A.M. Uranga, Nucl. Phys. B542 (1999) 112;

G. Aldazabal, L.E. Ibáñez and F. Quevedo, hep-th/9909172.

[20] Z. Kakushadze, Phys. Lett. B455 (1999) 120; hep-th/9904211; Phys. Lett. B559 (1999) 497.

[21] S. Hamidi and C. Vafa, Nucl. Phys. B279 (1987) 465;

L. Dixon, D. Friedan, E. Martinec and S. Shenker, Nucl. Phys. B282 (1987) 13.

[22] J. Polchinski, "TASI Lectures on D-branes", hep-th/9611050.

[23] J.A. Harvey, S. Kachru, G. Moore and E. Silverstein, hep-th/9909072. 
[24] N. Seiberg and E. Witten, JHEP 9909 (1999) 032.

[25] M. Bianchi, G. Pradisi and A. Sagnotti, Nucl. Phys. B376 (1992) 365;

Z. Kakushadze, Nucl. Phys. B512 (1998) 221;

M. Bianchi, Nucl. Phys. B528 (1998) 73;

E. Witten, JHEP 9802 (1998) 006;

Z. Kakushadze, G. Shiu and S.-H.H. Tye, Phys. Rev. D58 (1998) 086001;

Z. Kakushadze, Nucl. Phys. B444 (1999) 265;

C. Angelantonj, hep-th/9908064.

[26] C. Vafa, Nucl. Phys. B469 (1996) 403.

[27] M. Bershadsky, T. Pantev and V. Sadov, hep-th/9805056.

[28] A. Giveon and M. Roček, Nucl. Phys. B380 (1992) 128.

[29] P.S. Aspinwall, Phys. Lett. B357 (1995) 329.

[30] P.S. Aspinwall and M.R. Plesser, JHEP 9908 (1999) 001.

[31] A.K. Grant and Z. Kakushadze, hep-ph/9906556;

Z. Kakushadze, hep-th/9908016.

[32] Z. Kakushadze, Nucl. Phys. B551 (1999) 549.

[33] N. Arkani-Hamed and S. Dimopoulos, hep-ph/9811353;

Z. Berezhiani and G. Dvali, Phys. Lett. B450 (1999) 24;

T. Banks, A. Nelson and M. Dine, JHEP 9906 (1999) 014;

N. Arkani-Hamed and M. Schmaltz, hep-ph/9903417;

N. Arkani-Hamed, L. Hall, D. Smith and N. Weiner, hep-ph/9909326.

[34] F. Ardalan, hep-th/9910064. 